(REVIEW ARTICLE)

\title{
Precision medicine as a promising tool to empower research in healthcare industry
}

\author{
Sachdeva Mayank and Sinha Suhani * \\ Department of Pharmacy, School of Medical and Allied Sciences, GD Goenka University, Sohna Road, Gurugram-122103, \\ Haryana, India.
}

Publication history: Received on 18 July 2018; revised on 29 August 2018; accepted on 01 September 2018

Article DOI: https://doi.org/10.30574/gscbps.2018.4.3.0068

\begin{abstract}
Ever since the first human genome was sequenced in 2001, there has been growing investment and research interest in precision medicine aimed at targeting medicines for precise therapy. This concept uses genomic sequencing to diagnose rare disease conditions where conventional therapies have failed. Precision medicine is a treatment regime specifically tailored to have the highest chance of success and lowest risk of adverse effects. It promises three benefits first, to patients by improving efficacy of drugs and reducing adverse effects. Second, it saves the healthcare systems from the cost burden of treating adverse effects by reducing expenditure on ineffective drugs. Third, to pharmaceutical industry by enabling drug makers to select and evaluate drug candidates much more cheaply and quickly than is possible currently. It can be emphasized that, the "precision" of precision medicine relies on the availability of large amounts of high quality, diverse data (both clinical and genomic) to accurately and equitably inform diagnosis and treatment approaches. So, the quality of the outcomes facilitated will be determined and limited by the quality of the data used to inform them. This can be complemented and validated with an experienced physician, scientist and pathologist to realize its full potential and success. In this review, the methodologies adopted and recent advances in the precision medicine are summarized.
\end{abstract}

Keywords: Precision medicine; In silico; USFDA; Data mining; Algorithm; Artificial intelligence

\section{Introduction}

If realized completely in a pharmaceutical industry, precision or personalized medicines ensures targeted drug discovery and development processes employing artificial intelligence and existing datasets to perform better "in silico"'target identification [1]. They promise several advantages like; assist in improvement of food and drug administration (FDA) oversight of tests, drugs, and other technologies to support innovation while ensuring that these products are safe and efficacious [2]. Precision medicine enabled new partnerships of scientists in a wide range of specialties, as well as people from the patient advocacy community, universities, pharmaceutical companies, and others [3]. It provides better understanding of the underlying mechanisms by which various diseases occur as well as the roadmap to realizing the discovery of new chemical entities in a pharmaceutical company initiates with target selection, followed by the development of lead molecule for the target It provides better understanding of the underlying mechanisms by which various diseases occur as well as the roadmap to realizing the discovery of new chemical entities in a pharmaceutical company initiates with target selection, followed by the development of lead molecule for the target [4]. Once the molecule or new chemical entity is synthesized, in-vitro studies are performed to establish identification and validation for the route of synthesis, thereafter in-vivo studies are performed on patient derived pharmacological models or molecular profiles to establish proof of concept. This is followed by Clinical trials [5].

Precision medicine if realized to its full potential in hospitals, the treatments can be specifically adapted to the physical condition of the body, genetic, lifestyle and disease characteristics of a particular patient to not only determine what

\footnotetext{
${ }^{*}$ Corresponding author

E-mail address: suhani.sinha@gdgoenka.ac.in
} 
treatment should be administered, but also the particular dosage regimen for administration [6]. The identification of genetic or biological markers can be correlated with disease and the development of robustly validated diagnostics which can be used to screen for those markers. This technology facilitates in the development of high quality (and economically viable) screening protocols to identify high-risk individuals and enable prevention of disease rather than simply reactive treatment [7]. This technology could aid in design of new tools for building, analyzing, and sharing large sets of medical data as well as better integration of electronic health records (EHRs) in patient care, which will allow doctors and researchers to access medical data more easily [8].

\section{Methodology used in precision medicine}

Precision medicine is powered by patient data [9]. The health records and genetic codes of patients and healthy volunteers are vital, and help people to influence their own health care and the direction of research [10]. Hence accurate documentation is needed for useful outcomes.

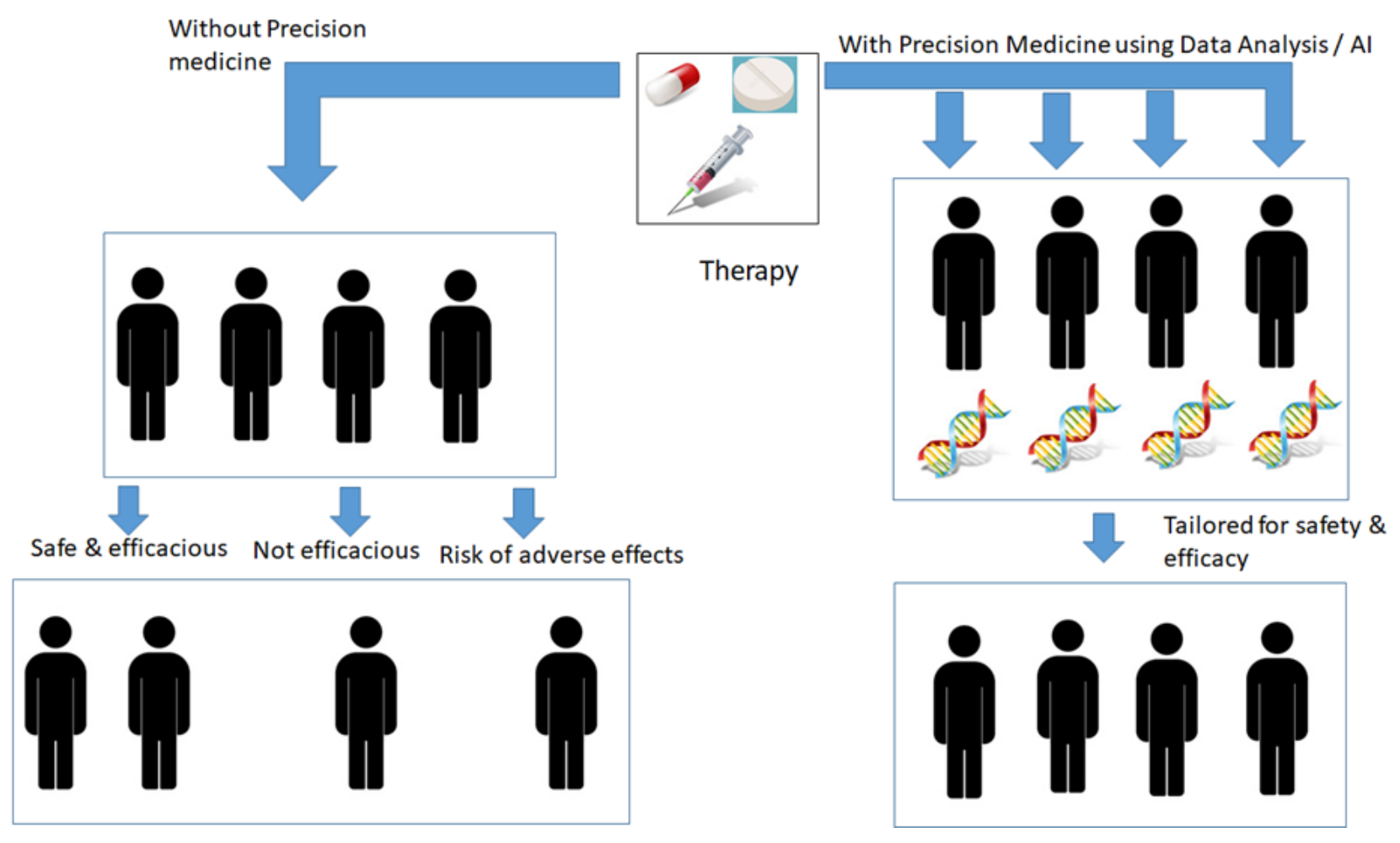

Figure 1 Representation of advantage of precision medicine

A pictographic representation of how precision medicine is advantageous over traditional concept is depicted above (Figure 1), wherein with same drug and dosage regimen being provided to patients for a particular indication uniformly to entire population or the drug being provided after careful data analysis of genome, age, lifestyle or ethnic origin using artificial intelligence is provided precisely to each individual (precision medicine) [11]. The former may or may not prove to be safe and efficacious to the population as a whole but the later has higher chances of safety and efficacy [12]. 


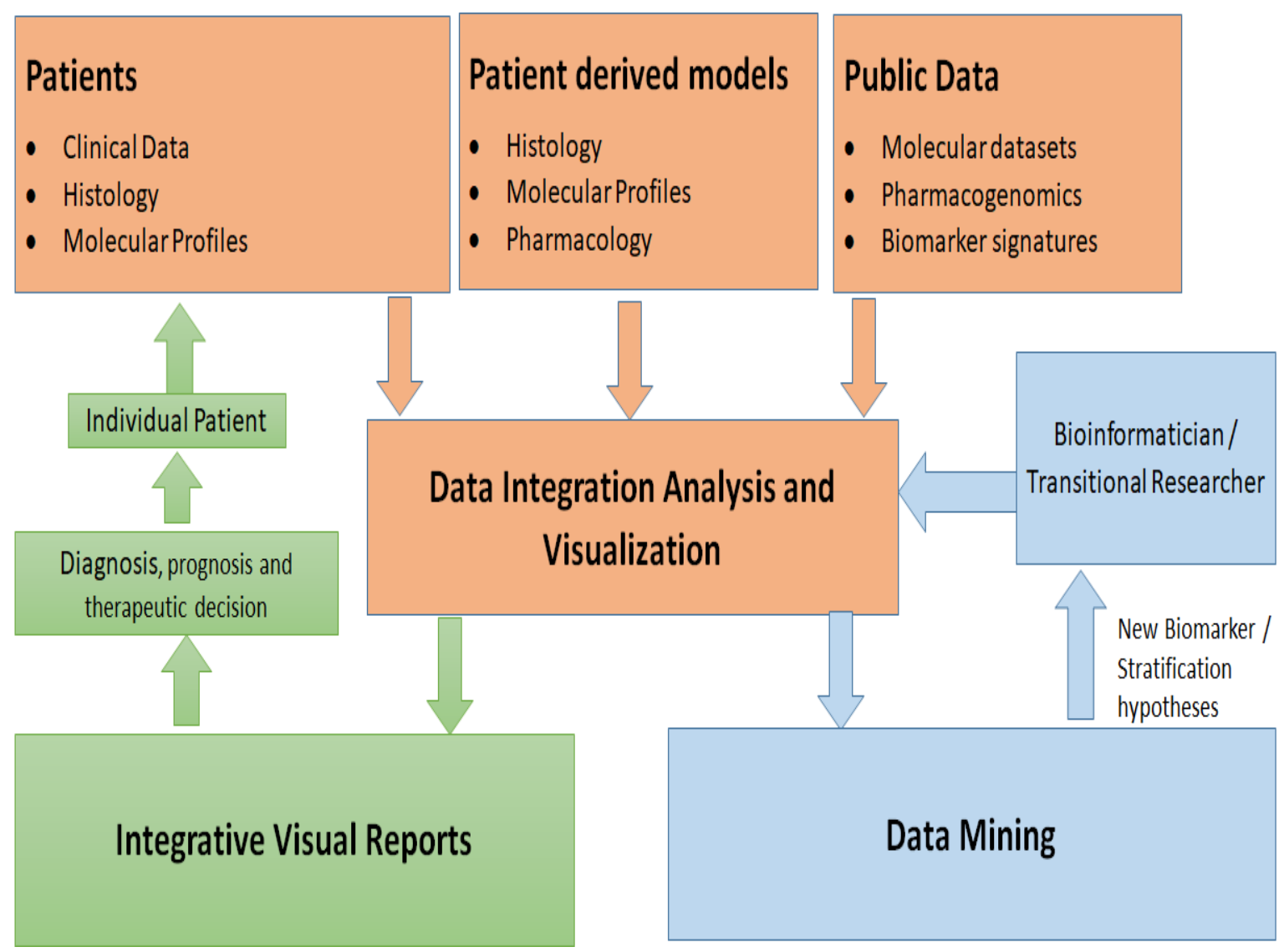

Figure 2 Representation of data integration and analysis for precision medicine

The graphical representation of patient data collection and analysis in precision medicine is shown in figure 2 . The raw data could be (i) sourced from patients, patient derived models and from public data bases (includes clinical data, histology, molecular profiles of patient collected through various diagnostics techniques); (ii) obtained through various patient derived models like histology, molecular profiles and pharmacology; (iii) extracted through molecular datasets, pharmacogenomics and biomarker signatures [13]. The entire data integration, analysis and visualization is then done by supercomputers using complex algorithms based on machine learning, artificial intelligence and then deep dive to yield integrative visual reports as outputs. These outputs leads to correct diagnosis, prognosis and therapeutic decision under the able guidance of expert physicians. Thus on one hand this process leads to individual patient receiving precise therapy and on the other hand the plethora of database allows the same therapy to be extended remotely to any patient for whom the outputs have been visualized to be same [13]. Thus it can be concluded that a substantial investment of time and resources in the present can unleash immense opportunity of electronically delivering expert medical opinion to remotest of areas and hence proving economical and time saving technique in future [14-15].

\section{Recent advances in precision medicine}

Despite various challenges and setbacks there has been significant advances in this area. Several gene therapies are edging closer to USFDA approvals and European authority approvals [16]. There has been several proof of evidence since its effective conceptualization and realization.

The Food and Drug Administration's approved the first chimeric antigen receptor T-cell (CAR-T) therapies (Novartis's Kymriah and Gilead/Kite's Yescarta). Kymriah, has just recently been granted approval for its second indication in relapsed/refractory large B-cell lymphoma, and both Kymriah and Yescarta are on the verge of approval in Europe [17]. Yescarta (axicabtagene ciloleucel, or axi-cel for short) has become the second CAR-T therapy to be approved in the US. The FDA has authorized its use to treat aggressive B-cell non-Hodgkin lymphoma in patients for whom at least two other types of treatments for their blood cancer failed $[18,19]$. Developed by US biotech Kite Pharma, Yescarta now belongs to Gilead. This makes Gilead the second to enter a whole new cancer market, right after Novartis, the developer of the first CAR-T therapy, Kymriah [19]. The approval of Yescarta is based on clinical trial results that are impressive considering the treatment is given to patients who don't respond to standard cancer treatments [20]. 
After a single infusion, $72 \%$ of the patients responded to the therapy, and $51 \%$ showed complete remission of cancer. However, there were three deaths due to side effects related to the treatment [19]. This is a first approval of a medicine by reference to the genetic features of the cancer as opposed to its site of origin. In this same vein, Loxo Therapeutics, a company specializing in the development of such targeted tumour agnostic treatments, has just released an abstract ahead of ASCO 2018, reporting a 69\% overall response rate in its phase I trial of LOXO-292 in 32 RET-fusion positive patients (including those with non-small cell lung cancer and papillary thyroid cancer), a result seen by many as the most impressive among those due to be announced at the leading oncology conference [7, 21].

The first in-human precision gene-editing trial (using zinc finger nucleases-Sangamo Therapeutics' treatment for Hunter Syndrome). CRISPR-based approaches are not far behind in the clinic, with CRISPR Therapeutics' Clinical Trial Application in Europe for a phase I/II of its lead pipeline product CTX001 for $\beta$-thalassemia approved in Q1 2018 and due to start in the second half of $2018[7,22,23]$.

At International Symposium on Biomedical Imaging, competitors created computational systems for detecting metastatic breast cancer in whole slide images of sentinel lymph node biopsies [24]. The winner algorithm had a $92.5 \%$ success rate. When a pathologist independently reviewed the same images, the success rate was $96.6 \%$. Combining the deep learning system's predictions with the human pathologist's diagnoses increased the pathologist's success rate to 99.5\%, an approximately $85 \%$ reduction in human error rate [7].

Britain's Benevolent AI is utilizing the technology to "analyze vast quantities of bioscience information" and identify compounds that are likely to effectively combat specific diseases without causing serious adverse effects [25]. Exscientia, which reports that its "knowledge-driven systems design millions of novel, project-specific compounds and pre-assess each for predicted potency, selectivity, ADME and other key criteria," signed a collaboration deal with GSK in 2017 [15]. Under the deal, Exscientia's AI enabled platform will be utilized in an effort to identify small molecules that can be used to treat up to ten targets selected by GSK [26]. Atomwise, says that its AI technology, called AtomNet, "helps predict the effectiveness of new drugs more rapidly" than conventional methods [15]. According to Atomwise, AtomNet researches small molecules with "unprecedented speed, accuracy and diversity." The company has reportedly partnered with Merck on malaria treatments [27].

\section{Conclusion}

Precision medicine has potential to yield huge benefits for heavily strained healthcare systems around the world. While there are major limitations in realizing precision medicine completely, there has been significant scientific discovery along this path. The existing challenges with budget and financial constraints for massive data collection and integration both clinical and genomic data into current computer systems should not deter the stakeholders at large for long term benefits. However precision medicine with its complex algorithms cannot function successfully without intervention of healthcare experts and the outcomes of data analysis should be complemented with expert clinical knowledge to validate the outcomes of algorithms. In short, to realize it completely, the institutions should communicate share knowledge with each other as understanding the diseases and how to treat them precisely will absolutely require a tremendous collaborative and integrative effort between research and medical institutions worldwide.

\section{Compliance with ethical standards}

\section{Acknowledgments}

Author is very much thankful to the G D Goenka University for their support in the preparation of this review.

\section{Disclosure of conflict of interest}

There is no conflict of interest.

\section{References}

[1] Collins FS and Varmus H. (2015). A new initiative on precision medicine. New England Journal of Medicine, 372(9), 793-5.

[2] Fackler JL and McGuire AL. (2009). Paving the way to personalized genomic medicine: steps to successful implementation. Current Pharmacogenomics and Personalized Medicine (Formerly Current Pharmacogenomics), 7(2), 125-32. 
[3] U.S. National Library of Medicine. What are some potential benefits of precision medicine ...? https://ghr.nlm.nih.gov/primer/precisionmedicine/potentialbenefits [Last accessed on 28 August, 2018].

[4] Strovel J, Sittampalam S, Coussens NP, Hughes M, Inglese J, Kurtz A, Andalibi A, Patton L, Austin C, Baltezor M and Beckloff M. (2016). Early drug discovery and development guidelines: for academic researchers, collaborators, and start-up companies.

[5] Hopkins AL. (2008). Network pharmacology: the next paradigm in drug discovery. Nature chemical biology, 4(11), 682.

[6] Hamburg MA and Collins FS. (2010). The path to personalized medicine. New England Journal of Medicine, 363(4), 301-4.

[7] Life Science Intellectual Property Review, The future of precision medicine part 1: challenges and opportunities. https://www.lifesciencesipreview.com/contributed-article/the-future-of-precision-medicine-part-1challenges-and-opportunities [Last accessed on 28 August, 2018]

[8] Palanisamy V and Thirunavukarasu R. (2017). Implications of Big Data Analytics in developing Healthcare Frameworks-A review. Journal of King Saud University-Computer and Information Sciences.

[9] Morgan AA, Mooney SD, Aronow BJ and Brenner SE. (2016). Precision medicine: data and discovery for improved health and therapy. In Biocomputing 2016: Proceedings of the Pacific Symposium, 243-248.

[10] Hodson R. (2016). Precision medicine. Nature, 537(7619), S49.

[11] Ashley EA. (2016). Towards precision medicine. Nature Reviews Genetics, 17(9), 507.

[12] Dolsten M and Søgaard M. (2012). Precision medicine: an approach to R\&D for delivering superior medicines to patients. Clinical and Translational Medicine, 1(1), 1-7.

[13] Mesko B. (2017). The role of artificial intelligence in precision medicine. Expert Review of Precision Medicine and Drug Development, 2(5), 239-241.

[14] Fleming N. (2018). How artificial intelligence is changing drug discovery. Nature, 557 (S57)

[15] Can Artificial Intelligence Help Drug Development? By Larry Ramer, Drug Patent Watch. https://www.drugpatentwatch.com/blog/can-artificial-intelligence-help-drug-development/ [Last accessed on 28 August, 2018].

[16] Maeder ML and Gersbach CA. (2016). Genome-editing technologies for gene and cell therapy. Molecular Therapy, $24(3), 430-46$.

[17] Yip A and Webster RM. (2018). The market for chimeric antigen receptor T cell therapies. Nature reviews. Drug discovery, 17(3), 161.

[18] FDA News Release, FDA approves CAR-T cell therapy ... B-cell lymphoma. https://www.fda.gov/newsevents/newsroom/pressannouncements/ucm581216.htm [Last accessed on 28 August, 2018].

[19] Medical, 'The FDA Approves a Second CAR-T Therapy, Cheaper than Novartis'. https://labiotech.eu/medical/yescarta-kymriah-car-t-therapy/ [Last accessed on 28 August, 2018]

[20] Medical, A Cure for Cancer? How CAR-T Therapy is Revolutionizing Oncology. https://labiotech.eu/features/cart-therapy-cancer-review/ [Last accessed on 28 August, 2018].

[21] Loxo Oncology Press release. https://ir.loxooncology.com/press-releases/loxo-oncology-announces-details-ofloxo-292-clinical-data-presentations-at-the-2018-american-society-of-clinical-oncology-asco-annual-meeting [Last accessed on 28 August, 2018].

[22] Shim G, Kim D, Park GT, Jin H, Suh SK and Oh YK. (2017). Therapeutic gene editing: delivery and regulatory perspectives. Acta Pharmacologica Sinica, 38(6), 738.

[23] Vertex, Investor Relations, News \& Events, CRISPR Therapeutics and Vertex Provide Update on FDA Review of Investigational New Drug Application for CTX001 for the Treatment of Sickle Cell Disease. https://investors.vrtx.com/news-releases/news-release-details/crispr-therapeutics-and-vertex-provideupdate-fda-review [Last accessed on 28 August, 2018].

[24] Wang D, Khosla A, Gargeya R, Irshad H and Beck AH. (2016). Deep learning for identifying metastatic breast cancer. arXiv preprint arXiv:1606.05718. 
[25] What we do? https://benevolent.ai/about-us/ [Last accessed on 28 August, 2018].

[26] Industry News / 2017 / GSK Signs \$43M Artificial Intelligence-Driven Drug Development Deal. https://www.pharmamanufacturing.com/industrynews/2017/gsk-signs-43m-artificial-intelligence-drivendrug-development-deal/ [Last accessed on 28 August, 2018].

[27] Atomwise news. https://www.atomwise.com/2015/12/02/introducing-atomnet-drug-design-withconvolutional-neural-networks/[Last accessed on 28 August, 2018].

\section{How to cite this article}

Sachdeva M and Sinha S. (2018). Precision medicine as a promising tool to empower research in healthcare industry. GSC Biological and Pharmaceutical Sciences, 4(3), 74-79. 